\title{
CONTRIBUIÇÕES PARA PROMOÇÃO DA SEGURANÇA DO PACIENTE E PREVENÇÃO DE QUEDAS
}

\author{
CONTRIBUTIONS TO THE PROMOTION OF PATIENT \\ SAFETY AND FALL PREVENTION
}

\section{CONTRIBUCIONES A LA PROMOCIÓN DE LA SEGURIDAD DEL PACIENTE Y LA PREVENCIÓN DE CAÍDAS}

\author{
Fabiele Aozane Cigana ${ }^{1}$ \\ Rosângela Marion da Silva ${ }^{2}$ \\ Carmem Lúcia Colomé Beck ${ }^{3}$ \\ Liliane Ribeiro Trindade ${ }^{4}$ \\ Ariane Naidon Cattani ${ }^{5}$ \\ Diogo Jardel Cigana ${ }^{6}$
}

Como citar este artigo: Cigana FA, Silva RM, Beck CLC, Trindade LR, Cattani AN, Cigana DJ. Contribuições para promoção da segurança do paciente e prevenção de quedas. Rev baiana enferm. 2019;33:e33280.

Objetivo: identificar a percepção de técnicas de enfermagem para promoção da segurança do paciente e prevenção de quedas em uma unidade de internação da clínica-médica. Método: estudo do tipo exploratório e descritivo, de abordagem qualitativa, realizado com técnicas de enfermagem, por meio de entrevista semiestruturada e observação sistemática não participante, no período de abril a junho de 2017. Para a análise dos dados, foi utilizada a Proposta Operativa de Minayo. Resultados: participaram 14 técnicas de enfermagem, que perceberam a necessidade de adequação das camas, da ocorrência de capacitações e do trabalho multiprofissional. Destacaram o quantitativo reduzido de profissionais da enfermagem e a ausência de familiar/acompanhante no cuidado como fatores que influenciam na segurança do paciente e prevenção de quedas. Conclusão: as técnicas de enfermagem identificaram a necessidade de adequação dos recursos físicos e humanos de enfermagem, a educação permanente e a participação do familiar/acompanhante para promoção da segurança do paciente e prevenção de quedas em uma unidade de internação da clínica-médica.

Descritores: Segurança do Paciente. Acidentes por Quedas. Enfermagem.

Objective: to identify the perception of nursing technicians to promote patient safety and fall prevention at a clinicalmedical inpatient unit. Method: exploratory and descriptive study, with qualitative approach, performed with nursing technicians, by means of semi-structured interviews and non-participant systematic observation, in the period from April to June 2017. For data analysis, the Operational Proposal of Minayo was used. Results: the participants were 14 nursing technicians, who realized the need for adequacy of beds, trainings and multiprofessional work. They

\footnotetext{
Enfermeira. Mestre em Enfermagem. Enfermeira Assistencial no Hospital Unimed Noroeste. ljuí, Rio Grande do Sul, Brasil. https://orcid.org/0000-000 I-6624-0566 Enfermeira. Doutora em Ciências. Professora da Universidade Federal Santa Maria. Santa Maria, Rio Grande do Sul, Brasil. cucasma@terra.com.br. https://orcid. org/0000-0003-3978-9654

Enfermeira. Doutora em Enfermagem. Professora da Universidade Federal Santa Maria. Santa Maria, Rio Grande do Sul, Brasil. https://orcid.org/0000-000 I-90601923

4 Enfermeira. Mestre em Enfermagem. Enfermeira na Estratégia de Saúde da Família. Prefeitura Municipal de Uruguaiana. Uruguaiana, Rio Grande do Sul, Brasil. https:// orcid.org/0000-0002-5906-4709

Enfermeira. Especialista em Saúde Mental. Universidade Federal de Santa Maria. Santa Maria, Rio Grande do Sul, Brasil. https://orcid.org/0000-0002-3365-9237

6 Enfermeiro. Enfermeiro Assistencial no Hospital de Caridade de ljú. ljuí, Rio Grande do Sul, Brasil. https://orcid.org/0000-000 I-839 I-2774
} 
highlighted the reduced number of nursing professionals and the absence of family/companion in care as factors that influence patient safety and fall prevention. Conclusion: the nursing technicians identified the need for adequacy of nursing buman and physical resources, continuing education and the participation of the family/companion to promote patient safety and fall prevention at a clinical-medical inpatient unit.

Descriptors: Patient Safety. Accidental Falls. Nursing.

Objetivo: identificar la percepción de técnicas de enfermería para promover la seguridad del paciente y la prevención de caídas en una unidad hospitalaria de la clinica médica. Método: estudio exploratorio y descriptivo, de enfoque cualitativo, realizado con técnicas de enfermeria, por medio de entrevistas semi-estructuradas y observación sistemática no participante, en el período de abril a junio de 2017. Para el análisis de datos, se utilizó la propuesta operacional de Minayo. Resultados: participaron en 14 técnicas de enfermería, que comprendieron la necesidad de adecuación de camas, capacitaciones y trabajo multiprofessional. Destacaron la cantidad reducida de profesionales de enfermería y la falta de familiares/acompañantes en el cuidado como factores que influyen en la seguridad del paciente y la prevención de caídas. Conclusión: las enfermeras técnicas identificaron la necesidad de adecuación de los recursos humanos y físicos de la enfermería, la educación permanente y la participación de la familial acompañante para promover la seguridad del paciente y la prevención de caídas en una unidad hospitalaria de la clínica médica.

Descriptores: Seguridad del Paciente. Accidentes por Caídas. Enfermería.

\section{Introdução}

A segurança do paciente tem sido o foco de discussões entre gestores e prestadores de serviços de saúde, com o intuito de melhorar a qualidade do cuidado na área da saúde. Dentre os cenários do cuidado de enfermagem, o ambiente hospitalar é aquele que proporciona uma complexidade de procedimentos e tratamentos aos assistidos. Por isso, esse é um espaço que potencializa a chance de ocorrer acidentes ${ }^{(1)}$. Os pacientes que se encontram em processo de restabelecimento da saúde estão vulneráveis às circunstâncias que podem resultar em dano desnecessário à saúde, como é o caso das quedas.

A queda prolonga o período de internação, com aumento dos custos, desconfortos físicos e psicológicos para o paciente ${ }^{(2)}$ e seus familiares. Ela caracteriza-se como um evento que faz com que o paciente termine de forma involuntária no chão ou em outro nível baixo, com presença ou ausência de lesões ${ }^{(3)}$.

A partir de 2013, o Programa Nacional de Segurança do Paciente ampliou o debate em instituições de saúde sobre o cuidado seguro. Isso, tem auxiliado no fortalecimento de ações que buscam melhorar a qualidade do cuidado, com a implantação de metas voltadas à segurança do paciente, especialmente aquelas relacionadas à prevenção de quedas $^{(4)}$. A ocorrência de quedas relaciona-se à perda do equilíbrio, a escorregões e tropeços e a desmaios, sendo o local da queda mais notificado a cama, seguida de quedas no banheiro e de cadeira ${ }^{(5)}$.

Estudo realizado com 228 idosos em um hospital de ensino de grande porte aponta que os pacientes sofrem queda ao realizar alguma atividade que pode ser considerada simples, como levantar do leito $(33,3 \%)$ e caminhar até o banheiro (44,4\%), sendo necessária uma atenção diferenciada da equipe de saúde, devido à vulnerabilidade que esses apresentam quanto aos riscos decorrentes da internação, particularmente o risco de quedas ${ }^{(6)}$. Nesse contexto, é relevante a atuação da equipe, especialmente a de enfermagem, na prevenção de quedas no ambiente hospitalar, o que implica um diálogo permanente na construção de propostas para a educação em saúde alicerçadas na singularidade do cuidado.

Desse modo, justifica-se este estudo por entender-se que, para aprimorar a segurança do paciente, é preciso estar em constante atualização profissional, além de conhecer e entender as especificidades da instituição. Dentre as profissões atuantes nos serviços de saúde, a equipe 
de enfermagem está em contato direito com o paciente em todos os turnos de trabalho. Neste sentido, é importante analisar o cenário e a organização de trabalho, como a equipe desenvolve suas atividades e a complexidade dessas ações, o que pode favorecer a implantação de estratégias para práticas assistenciais mais seguras e contribuir para sinalizar comportamentos a serem evitados ${ }^{(7)}$.

Assim, considerando que a organização do trabalho está diretamente relacionada a ações seguras, e essas promovem cuidado com qualidade nas instituições hospitalares, questiona-se: Quais as percepções de técnicas de enfermagem para a promoção da segurança do paciente e prevenção de quedas em uma unidade de internação da clínica-médica? O objetivo foi identificar a percepção de técnicas de enfermagem para promoção da segurança do paciente $\mathrm{e}$ prevenção de quedas em uma unidade de internação da clínica-médica.

\section{Método}

Estudo do tipo exploratório e descritivo, de abordagem qualitativa. Foi realizado com técnicas de enfermagem de um hospital localizado em um município do estado do Rio Grande do Sul no período de abril a junho de 2017. A instituição contava com 112 leitos e era considerada referência em média e alta complexidade de 52 municípios da sua região. A unidade selecionada para a realização do estudo foi a de internação clínica-médica, com 35 leitos, local em que o Núcleo de Segurança do Paciente da instituição implantou inicialmente os protocolos de identificação do paciente, segurança na prescrição, no uso e na administração de medicamentos e prevenção de quedas.

$\mathrm{Na}$ unidade atuavam 41 trabalhadores de enfermagem (4 enfermeiros e 37 técnicos de enfermagem), divididos nos turnos da manhã (13 trabalhadores), tarde (11 trabalhadores) e noite (17 trabalhadores). Estipulou-se como critérios de inclusão ser técnico de enfermagem e possuir, no mínimo, seis meses de experiência na unidade. O critério de exclusão foi estar afastado do trabalho por licença de qualquer natureza. Foi realizado sorteio dos participantes por turnos de trabalho. Optou-se por não incluir os enfermeiros no estudo, tendo em vista o quantitativo, pois poderia favorecer a identificação nos turnos de trabalho.

Para a coleta de dados, utilizou-se a entrevista semiestruturada e a observação sistemática não participante. Todas as etapas foram realizadas por uma pesquisadora, estudante de pós-graduação em enfermagem, nível mestrado. A entrevista foi realizada individualmente, nos turnos da manhã, tarde e noite, em uma sala reservada, localizada nas dependências da instituição, espaço que garantiu a privacidade do participante. Seguiu um roteiro previamente elaborado, que contemplava dados sociolaborais (sexo, idade, estado civil, filhos, outro emprego, escolha pelo turno de trabalho, tempo de trabalho na categoria) e questões norteadoras, que buscaram gerar conhecimentos acerca das ações desenvolvidas para promover a segurança do paciente na instituição. A entrevista foi audiogravada em gravador digital, após o consentimento do participante, tendo duração média de 25 minutos cada.

O término dessa etapa ocorreu quando as informações tornaram-se reincidentes, não resultando em novos achados, o que atende ao critério de saturação amostral $^{(7)}$. Para preservar o anonimato, utilizou-se a letra $\mathrm{P}$ (participante), seguida de um número arábico correspondente à ordem da realização da entrevista $(1,2,3 .$.$) e a inicial do turno de$ trabalho (manhã - M; tarde - T; noite - N).

A observação sistemática não participante foi realizada em todos os turnos, totalizando nove, com duração média de 4 horas cada, perfazendo um total de 36 horas. Foram observadas: dinâmica de trabalho em equipe, organização/ divisão do trabalho, comportamento da equipe diante de um problema ou atividade não prevista e ocorrência de eventos adversos. Foram registradas em um diário de campo e identificadas pela sigla NO (notas de observação), seguida da data correspondente e da inicial do turno. Foram lidas exaustivamente na busca de convergência com os dados das entrevistas. 
Para a análise dos dados, foi utilizada a Proposta Operativa de Minayo, caracterizada por dois momentos interpretativos. O primeiro, constituído pelo mapeamento do campo das determinações fundamentais, que se refere ao contexto histórico do grupo social em foco. O segundo é a convergência com os fatos empíricos, quando se encontra o sentido, a lógica interna, as projeções e as interpretações nos relatos dos participantes. Para operacionalizar esse segundo momento, realiza-se a ordenação e classificação dos dados em quatro etapas: leitura horizontal, primeiro contato com os dados obtidos; leitura transversal, gerando as categorias; análise final, em que os dados obtidos são discutidos teoricamente; e relatório, que finaliza a apresentação dos resultados da pesquisa ${ }^{(8)}$.

Este estudo foi aprovado pelo Comitê de Ética em Pesquisa, sob o Parecer n. 1.982.855. Atende aos preceitos da Resolução n. 466/12, do Conselho Nacional de Saúde. Todos os participantes assinaram o Termo de Consentimento Livre e Esclarecido. Destaca-se que este manuscrito obedece aos critérios consolidados para relatórios de estudos qualitativos ${ }^{(9)}$.

\section{Resultados}

Participaram do estudo 14 técnicas de enfermagem, todas do sexo feminino, com idade entre 20 e 39 anos; 71,72\% tinham filhos e metade possuía companheiro; tinham tempo de trabalho até cinco anos na categoria e não possuíam outro vínculo empregatício; 57,15\% escolheram o turno de trabalho.

Em relação à segurança do paciente e à prevenção de quedas, instituída em protocolo, as participantes relataram que um olhar diferenciado referente aos cuidados de enfermagem pode favorecer a promoção de práticas seguras.

Só porque o paciente caminhava, antes não se levantavam as grades. Mas o paciente pode dormir, pode ter uma queda. Agora está bem mais tranquilo. Questão de ir ao banheiro, eles não arriscam ir sozinhos, eles chamam. Isto deixa a gente bem mais tranquilo. Tu sabe que não vai acontecer nada. (P1, M).

Era uma coisa tempos atrás, a gente não tinha essa visão. A gente pensava: se o paciente está bem, lúcido, não imaginava que ele ia cair, mas aconteceram casos de pacientes que caíram da cama. (P13, N).
A adequação das camas foi sinalizada como recurso que pode impactar na segurança do paciente.

\begin{abstract}
Segurança física, assim, melhoramento de camas, por exemplo [...] os colchões, que vários pacientes já reclamaram que os colchões são duros, que as camas são muito altas. E realmente as camas são altas. As que têm três manivelas, você pode baixar até o máximo, que daí fica melhor, principalmente para os idosos, que não têm muita força nas pernas, então é mais fácil para eles saírem da cama [...] poderia ter umas macas diferentes. São muito estreitinhas para um paciente maior, pode acontecer alguma coisa. (P4, T).
\end{abstract}

As participantes mencionaram que o trabalho integrado da enfermagem com outras profissões da área da saúde, como a fisioterapia, pode auxiliar no planejamento da assistência e na prevenção de quedas.

Quando forem feitos os planejamentos, como da fisioterapia ou da enfermagem, deveria existir um representante de cada, porque a gente trabalha todo mundo em equipe. Quando existir uma mudança de planejamento dentro das rotinas, será que é viável para fisioterapia? Será que é viável para a enfermagem? Será que é viável para higienização? Um pensar no outro, para ambos pensarem juntos, para poderem planejar juntos. (P8, M).

Observou-se que existe o envolvimento de outros trabalhadores, como a fisioterapia, para auxilio na retirada do paciente do leito. Percebeu-se que a fisioterapia realiza avaliação para o risco de queda e comunica à equipe de enfermagem se o risco for identificado. Existe uma boa comunicação e ajuda entre a enfermagem e a fisioterapia. (NO, 27/5/2017, M).

Outro fator apontado pelas técnicas de enfermagem, que pode contribuir para minimizar os eventos adversos, é a educação permanente, referida como capacitações.

As capacitações, com certeza são de suma importância para o paciente, para a equipe de enfermagem, para os familiares [...] que não envolve só o paciente, mas sim o todo. A gente se sente valorizado. $(\mathrm{P} 7, \mathrm{~N})$.

Mas uma coisa que eu acho que está faltando boje na unidade, durante a noite, que não está sendo mais cobrado e não está sendo mais feito e tinha antigamente, é aqueles cursinbos da madrugada: "Pessoal, vem aqui, vamos ver o carrinho, vamos abrir, vamos olhar."; "Olha aqui, veio uma bomba nova!" Isso se perdeu. Isso eu vejo que não tem mais. Nós tínhamos enfermeiras que nos levavam de madrugada para os quartos e lá treinava massagens, treinava simulação de parada! Tinha "Fulano de tal é pro carrinho, a outra é pra massagem, o outro, pra ligar pro médico." Tinha na época, quando eu entrei. (P10, N).

Eu fui a uma capacitação de segurança do paciente sobre pulseira, administração de medicamentos, dietas, dos riscos de quedas, da plaquinha de identificação. Até deveria ter mais, porque isso é uma rotina nossa. Nós lidamos com a vida das pessoas todos os dias. Deveria ter mais seguido capacitações, pois temos bastante gente nova e, mesmo tendo acompanhamento da enfermagem, 
os novos que entram, nós somos orientados a seguir o que a enfermeira diz. Mas é uma coisa tão grande que a pessoa não tem noção. A pessoa está entrando em uma profissão nova, não tem noção. É muito grande a questão de segurança do paciente. (P8, M).

Foi destacado pelas participantes que o quantitativo reduzido de profissionais pode sobrecarregar as trabalhadoras e comprometer a segurança do paciente.

Tem dias que a gente consegue dar um pouco mais de atenção ao paciente e tem outros dias que mal a gente consegue trocar palavras, e cada dia é um dia. (P2, T).

Observou-se, no posto de enfermagem, no perído noturno, menos trabalhadores em comparação aos outros turnos de trabalbo. Na divisão do quadro de pacientes, os trabalhadores ficam com maior quantitativo de pacientes, quando comparado aos turnos da manhã e tarde. (NO, 29/5/2017, N).

A ausência de familiar/acompanhante nos quartos, foi referida como fator que pode influenciar as práticas seguras.

Tem paciente que fica sozinho, e isto teria que ter uma lei, que teria que ficar um acompanbante. Porque tu não tem como ficar 6 horas do teu plantão com aquele paciente e, às vezes, aquele paciente exige muito de ti, por estar sozinho. Eu acho que isso deveria ser uma orientação já para ofamiliar. (P1, M).

Notou-se que os trabalhadores procuram esclarecer in formações solicitadas pelos pacientes e familiares, sendo prestativos. Percebeu-se que os trabalhadores desenvolvem uma atenção diferenciada aos pacientes, por meio de conversas, apoio, estabelecem vinculo com pacientes e familiares. (NO, 12/6/2017, T); (NO, 22/5/2017, N); (NO, 27/5/2017, M); (NO, 29/5/2017, N).

No posto de enfermagem, o técnico de enfermagem relatou ao enfermeiro da unidade a ocorrência de uma queda de um paciente. O técnico relatou que deixou as grades do leito erguidas, porém o paciente saiu do leito sem auxílio da enfermagem, sofrendo uma queda. No relato, o técnico de enfermagem menciona que o familiar auxiliou até o deslocamento ao banbeiro, porém o paciente apresentou tontura, sofrendo queda no local, ao sentar-se no vaso sanitário. O enfermeiro foi até o quarto avaliar o paciente e comunicou ao médico assistente. Este fato foi notificado pelo enfermeiro na caixa de notificações existente na unidade, por meio de um formulário de descrição da notificação. (NO, 12/06/2017, T).

\section{Discussão}

Os resultados apontaram que as técnicas de enfermagem percebiam situações do processo de trabalho que eram passíveis de transformação, como adequação dos recursos físicos e o trabalho em equipe, pois eram instrumentos que preveniam quedas e promoviam a segurança do paciente. Além disso, havia necessidade de disseminar a cultura de segurança nos serviços de saúde, sendo essa uma das metas institucionais ${ }^{(10)}$.

A articulação conjunta com trabalhadores de outras áreas possibilita a diminuição de incidentes com interferência na segurança do paciente $^{(11)}$. Entende-se que a atuação multiprofissional pode auxiliar na prevenção de quedas, quando há comunicação entre trabalhadores de diferentes profissões da área da saúde. As equipes de enfermagem e fisioterapia têm direcionado cuidados em conjunto na prevenção de quedas, contribuindo para a segurança do paciente.

As capacitações contribuem para práticas seguras, mas essa iniciativa deve partir de ações pactuadas coletivamente com a gerência e a administração do hospital. A participação do enfermeiro nessa prática é fundamental, especialmente na organização desses momentos com a equipe e para identificar as necessidades de temas a serem trabalhados, pois a prática de capacitações junto aos trabalhadores torna a assistência mais segura, influenciando diretamente na segurança do paciente e do trabalhador ${ }^{(12)}$. Uma técnica de enfermagem mencionou o papel e a importância da enfermeira em estimular algumas práticas de segurança do paciente, como capacitação no local de trabalho.

Em um estudo sobre cultura de segurança do paciente, participantes também expressaram o desejo de aperfeiçoar sua formação profissional para proporcionar uma assistência segura. Tem-se a participação em treinamentos, capacitações, aperfeiçoamentos, grupos de estudo, acompanhamento dos funcionários novos, reuniões entre técnicos em enfermagem, enfermeiros e subgerência ${ }^{(13)}$, reiterando a importância da incorporação de programas de educação permanente com os trabalhadores nos estabelecimentos de saúde.

Realizar ações educativas com a equipe de enfermagem é uma estratégia que pode ser utilizada para motivá-la para o trabalho. A educação permanente é considerada uma prática para alcançar a redução de danos ao paciente, pois incentiva o compartilhamento de conhecimentos e experiências. Possibilita ao profissional de saúde 
espaço para discutir e compartilhar situações cotidianas, diminuindo a sobrecarga de trabalho e instrumentalizando-o a respeito de experiências provenientes da ocorrência de eventos adversos. Auxilia na progressão de uma cultura de segurança do paciente mais significativa aos trabalhadores $^{(14)}$, o que faz dela importante prática, que pode promover a mudança de comportamento desses e colaborar nas ações seguras.

A prática de educação permanente permite refletir sobre as diversas questões presentes no cotidiano do trabalho ${ }^{(15)}$. No presente estudo, notou-se a necessidade de haver seguidamente capacitações, inclusive sobre o risco de quedas, justificadas pela atuação direta da equipe de enfermagem nos cuidados com o paciente. Além disso, a realização de capacitações permitirá aproximação com a temática de segurança do paciente.

Identificou-se, por meio da Nota de Observação, que o quantitativo de trabalhadores é uma situação que sugere sobrecarga para os trabalhadores, pois há desproporção entre o número de trabalhadores de enfermagem e de pacientes. Este fato pode favorecer o aumento da incidência de eventos adversos. Isto corrobora achados de estudo chinês, que constatou a relação direta entre a melhoria da segurança do paciente no cenário hospitalar e o dimensionamento adequado da equipe de enfermagem ${ }^{(16)}$.

A sobrecarga, observada nos relatos dos trabalhadores, quando referiram que havia dias em que não conseguiam dar atenção necessária ao paciente, pode interferir no bem-estar desses e causar estresse, fatores que também estão associados à ocorrência de danos aos pacientes. Pode, ainda, implicar no tempo dispensado ao cuidado, elemento que tem relação com as práticas seguras, pois a redução do tempo de vigilância e de auxílio nas atividades dos pacientes aumenta o risco de quedas e demais incidentes.

Estudo inglês aponta evidências de o bem-estar e o estresse excessivo estarem associados à segurança do paciente, isto é, os erros autorreferidos estão associados às pessoas, com prejuízo do bem-estar ${ }^{(17)}$. A sobrecarga de trabalho exige esforços do trabalhador para manter a qualidade dos cuidados ofertados. A participação do familiar/acompanhante no processo de cuidar é fator importante para minimizar essa carga, além de oportunizar o compartilhamento das informações.

O familiar/acompanhante, nesse processo, pode ser um dos agentes promotores da segurança do paciente, tendo em vista que os cuidados para prevenção de queda envolvem a orientação ao paciente e ao familiar, no intuito de estimular sua participação no cuidado ${ }^{(12)}$. Entende-se que situações em que o paciente permanece internado sem a presença de familiar/ acompanhante poderão aumentar o risco de quedas e comprometer a sua segurança.

Assim, assinala-se a importância da permanência do familiar/acompanhante junto ao paciente, pois sua presença é um dos fatores que auxilia na prevenção de eventos adversos. Promover o seu envolvimento permite integrá-lo no cuidado de acordo com sua capacidade. Essa aproximação possibilita uma prática colaborativa entre os familiares/acompanhantes e os trabalhadores de enfermagem, contribuindo, dessa forma, para a promoção da cultura de segurança ${ }^{(18)}$.

Vale ressaltar que, por mais importante que seja a presença dos familiares/acompanhantes no período de internação, é dever da equipe de saúde a promoção da segurança do paciente e a prevenção de quedas. A prática da enfermagem deve estar centrada no cuidado, com respaldo no conhecimento científico, no diálogo e no estabelecimento de relações interpessoais saudáveis com vistas à promoção de um ambiente seguro ${ }^{(19)}$.

Além disso, a equipe de saúde é responsável por notificar a ocorrência de eventos adversos, como quedas. $\mathrm{Na}$ instituição onde foi realizada a pesquisa, as notificações recebidas pelo Núcleo de Segurança do Paciente eram discutidas em reuniões mensais, na busca de estratégias que tornassem as ações mais seguras. Quando necessário, elas eram informadas no Sistema de Notificações em Vigilância Sanitária (Notivisa). Destaca-se que os pacientes e seus familiares também podem realizar as notificações, contribuindo para a tomada de decisões por parte do Núcleo de Segurança do Paciente. 
Para o estabelecimento de medidas de prevenção para eventos adversos, como as quedas, algumas práticas recomendadas incluem a utilização de pisos antiderrapantes, instalação de lâmpadas de segurança nos banheiros e corredores, uso de dispositivos de auxílio para deambulação com supervisão, instalação de barras de apoio no banheiro e no chuveiro, manutenção de grades no leito do paciente e reforço de orientações para os acompanhantes dos pacientes com diagnóstico de risco para quedas ${ }^{(1)}$. As configurações físicas, materiais e organizacionais dos serviços de saúde, como instalações, equipamentos, recursos financeiros, qualificação e quantidade dos recursos humanos, relacionam-se com a qualidade do cuidado em saúde ${ }^{(20)}$.

As limitações do estudo estão relacionadas ao método adotado, pois o estudo descritivo restringe os resultados ao local investigado, não permitindo generalizar para outros contextos. Outro fator considerado como limitante foi a não inclusão, como participantes, de outros profissionais da saúde, tendo em vista que a segurança do paciente é tema transversal para todas as profissões. Apesar disso, a análise ofereceu informações que possibilitariam planejar práticas mais seguras relacionadas à prevenção de quedas e consequentemente à diminuição da ocorrência desse evento adverso, além de possibilitar o planejamento e a implementação de ações, pelo Núcleo de Segurança do Paciente e pelas gerências dos serviços de enfermagem, que tornem o período de hospitalização mais seguro.

\section{Conclusão}

A adequação dos recursos físicos e humanos de enfermagem, como trabalho multiprofissional, e o dimensionamento adequado de profissionais de enfermagem, a educação permanente e a participação do familiar/acompanhante no cuidado foram percepções relatadas pelas técnicas de enfermagem de uma unidade de internação da clínica médica que auxiliavam na promoção da segurança do paciente e na prevenção de quedas.

Espera-se que esses resultados possam contribuir para a comunidade científica, graduandos em enfermagem, trabalhadores docentes, equipe de enfermagem e Núcleos de Segurança do Paciente, para que ações sejam definidas coletivamente e constituam-se em práticas seguras. A segurança do paciente representa desafio para a excelência da qualidade no serviço de saúde, contudo os resultados apresentados podem contribuir para uma melhor assistência ao paciente no que se refere ao cuidado seguro.

\section{Colaborações:}

1. concepção, projeto, análise e interpretação dos dados: Fabiele Aozane Cigana, Ariane Naidon Cattani e Rosângela Marion da Silva;

2. redação do artigo e revisão crítica relevante do conteúdo intelectual: Fabiele Aozane Cigana, Rosângela Marion da Silva e Carmem Lúcia Colomé Beck;

3. aprovação final da versão a ser publicada: Rosângela Marion da Silva, Liliane Ribeiro Trindade e Diogo Jardel Cigana.

\section{Referências}

1. Rede Brasileira de Enfermagem e Segurança do Paciente. Estratégias para a segurança do paciente: manual para profissionais da saúde. Porto Alegre: Edipucrs; 2013. 132 p.

2. Kim K, Jung HK, Kim CO, Kim SK, Cho HH, Kim DY, et al. Evidence-based guidelines for fall prevention in Korea. Korean J Intern Med [Internet]. 2017 [cited 2018 Jun 10];32(1):199-210. Available from: https://www.ncbi.nlm.nih.gov/pmc/articles/ PMC5214733/

3. Health Services Research Group University of Newcastle. Australasian Clinical Indicator Report. 15th ed. Newcastle; 2014. 132 p.

4. Brasil. Ministério da Saúde. Agência Nacional de Vigilância Sanitária. Resolução da Diretoria Colegiada $n^{-}$36, de 25 de julho de 2013. Institui ações para a segurança do paciente em serviços de saúde e dá outras providências [Internet]. Brasília (DF); 2013 [cited 2018 Jan 13]. Available from: http://portal.anvisa.gov.br/ documents/10181/2871504/RDC_36_2013_COMP. pdf/36d809a4-e5ed-4835-a375-3b3e93d74d5e 
5. Brasil. Ministério da Saúde. Agência Nacional de Vigilância Sanitária. Boletim Segurança do Paciente e Qualidade em Serviços de Saúde: Incidentes Relacionados à Assistência à Saúde [Internet]. Brasília (DF); 2016 [cited 2018 Jan 12]. Available from: https://www20.anvisa.gov.br/ segurancadopaciente/index.php/publicacoes/ category/boletins-estatisticos

6. Vaccari É, Lenardt MH, Willig MH, Betiolli SE, Andrade LAS. Segurança do paciente idoso e o evento queda no ambiente hospitalar. Cogitare Enferm [Internet]. 2016 [cited 2018 Feb 20]; 21(esp):1-9. Available from: https://revistas.ufpr.br/ cogitare/article/view/45562/pdf

7. Siqueira CL, Ferreira KM, Souza TC, Feldman LB. Sentimentos experimentados por equipes de enfermagem acerca dos erros de medicação. Cogitare Enferm [Internet]. 2016 [cited 2018 Jun 16];21(esp.):1-10. Available from: https://revistas. ufpr.br/cogitare/article/view/45411

8. Minayo MCS. O desafio do conhecimento: pesquisa qualitativa em saúde. 14a ed. São Paulo: Hucitec; 2014.

9. Tong A, Sainsbury P, Craig J. Consolidated criteria for reporting qualitative research (COREQ): a 32item checklist for interviews and focus groups. Int J Qual Health Care [Internet]. 2007 [cited 2017 Sep 30];19(6):349-57. Available from: https://academic. oup.com/intqhc/article/19/6/349/1791966

10. Dias JD, Mekaro KS, Tibes CMS, Zem-Macarenhas SH. Compreensão de enfermeiros sobre segurança do paciente e erros de medicação. REME [Internet]. 2014 [cited 2017 Dec 10];18(4): 866-73. Available from: http://www.reme.org.br/artigo/detalhes/969

11. Pasa TS, Magnago TSBS, Urbanetto JS, Baratto MAM, Morais BX, Carollo JB. Avaliação do risco e incidência de quedas em pacientes adultos hospitalizados. Rev Latino-Am Enfermagem. 2017;25:e2862. DOI: 10.1590/1518-8345.1551.2862

12. Aruto GC, Lanzoni GMM, Meirelles BHS. Melhores práticas no cuidado à pessoa com doença cardiovascular: interface entre liderança e segurança do paciente. Cogitare Enferm [Internet]. 2016 [cited 2018 Apr 15];21(esp.):1-9. Available from: https://revistas.ufpr.br/cogitare/article/ view/45648

13. Fermo VC, Radunz V, Rosa LM, Marinho M. Professional attitudes toward patient safety culture in a bone marrow transplant unit. Rev
Gaúcha Enferm. 2016;37(1):e55716. DOI: 10.1590/1983-1447.2016.01.55716

14. Wegner W, Silva SC, Kantorski KJC, Predebon CM, Sanches MO, Pedro ENR. Education for culture of patient safety: Implications to professional training. Esc Anna Nery. 2016;20(3):e20160068. DOI: $10.5935 / 1414-8145.20160068$

15. Figueiredo ML, Oliveira e Silva CS, Brito MFSF, D'Innocenzo M. Analysis of incidents notified in a general hospital. Rev Bras Enferm. 2018;71(1):111-9. http://dx.doi.org/10.1590/0034-7167-2016-0574

16. Liu X, Zheng J, Liu K, Baggs JG, Liu J, Wu Y, et al. Hospital nursing organizational factors, nursing care left undone, and nurse burnout as predictors of patient safety: A structural equation modeling analysis. Int J Nurs Stud. 2018;20(86):82-9. DOI: 10.1016/j.ijnurstu.2018.05.005

17. Hall LH, Johnson J, Watt I, Tsipa A, O'Connor DB. Healthcare Staff Wellbeing, Burnout, and Patient Safety: A Systematic Review. PLoS One. 2016; 11(7):e0159015. DOI: 10.1371/journal.pone. 0159015

18. Passos SSS, Henckemaier L, Costa JC, Pereira A, Nitschke RG. Cuidado quotidiano das famílias no hospital: como fica a segurança do paciente? Texto contexto-enferm. 2016;25(4):e2980015. DOI: 10.1590/0104-07072016002980015

19. Cavalcante AKCB, Rocha RC, Nogueira LT, Avelino FVSD, Rocha SS. Cuidado seguro ao paciente: contribuições da enfermagem. Rev cuba enferm [Internet]. 2015 [cited 2018 Nov 6];31(4). Available from: http://www.revenfermeria.sld.cu/ index.php/enf/article/view/907

20. Moore L, Lavoie A, Bourgeois G, Lapointe J. Donabedian's structure-process-outcome quality of care model: Validation in an integrated trauma system. J Trauma Acute Care Surg. 2015;78(6):116875. DOI: $10.1097 /$ TA.0000000000000663

Recebido: 26 de agosto de 2019 Aprovado: 2 de novembro de 2019 Publicado: 4 de fevereiro de 2020 
A Revista Baiana de Enfermagem utiliza a Licença Creative Commons - Atribuição-NãoComercial 4.0 Internacional. https://creativecommons.org/licenses/by-nc/4.0/ Este artigo é de acesso aberto distribuído sob os termos da Licença Creative Commons (CC BY-NC). Esta licença permite que outros remixem, adaptem e criem a partir do seu trabalho para fins não comerciais. Embora os novos trabalhos tenham de lhe atribuir o devido crédito e não possam ser usados para fins comerciais, os usuários não têm de licenciar esses trabalhos derivados sob os mesmos termos. 